\title{
Changes in Biomarkers after Initial Periodontal Treatment in Gingival Crevicular Fluid from Patients with Chronic Periodontitis Presenting with Drug-Induced Gingival Overgrowth
}

\author{
Taro Shimizu ${ }^{1,2}$, Takehiko Kubota ${ }^{1 *}$, Masanori Iwasaki ${ }^{3}$, Toshiya Morozumi ${ }^{1}$, \\ Hiromasa Yoshie ${ }^{1}$ \\ ${ }^{1}$ Division of Periodontology, Department of Oral Biological Science, Niigata University Graduate School of \\ Medical and Dental Sciences, Niigata, Japan \\ ${ }^{2}$ Oral Implant Clinic, Niigata University Medical and Dental Hospital, Niigata, Japan \\ ${ }^{3}$ Division of Preventive Dentistry, Department of Oral Biological Science, Niigata University Graduate School of \\ Medical and Dental Sciences, Niigata, Japan \\ Email:"kubota@dent.niigata-u.ne.jp
}

Received 18 January 2016; accepted 26 February 2016; published 29 February 2016

Copyright $(\underset{2016}{ } 2 \mathrm{by}$ authors and Scientific Research Publishing Inc.

This work is licensed under the Creative Commons Attribution International License (CC BY). http://creativecommons.org/licenses/by/4.0/

(c) (i) Open Access

\section{Abstract}

Calcium channel blocker-induced gingival overgrowth (CCB-GO) is increasing in elderly patients who have been prescribed medication for hypertension for years. The purpose of the present study was to analyze the comprehensive protein expression levels of candidate biomarkers in gingival crevicular fluid (GCF) from CCB-GO patients. Eleven GO patients (10 males and one female, mean \pm SD: age: $64.4 \pm 14.0$ years) who had been systemically prescribed CCBs, either amlodipine or nifedipine, for hypertension for at least 12 months were recruited. Before (baseline) and 4 weeks after initial periodontal treatments, subgingival plaque and GCF samples were taken from two sites per patient: sites affected by CCB-GO and chronic periodontitis. Measurement of clinical parameters and quantitative analysis of periodontopathic bacteria using real-time PCR were performed. Biomarkers/cytokines in GCF were examined using multiplex bead immunoassays. The Mann-Whitney $U$ test was used to compare the collected data between groups. The correlations between pairs of biomarkers were assessed using the Spearman correlation relationship. Levels of two of the 14 biomarkers, interleukin (IL)-1 $\beta$ and transforming growth factor (TGF)- $\beta$, were significantly decreased in CCB-GO sites after initial periodontal therapy. The intragroup comparison

\footnotetext{
${ }^{*}$ Corresponding author.
} 
at baseline showed that counts of Treponema denticola in the GO group were significantly higher than those in the chronic periodontitis group $(P<0.05)$. The results confirmed that initial periodontal therapy effectively reduces pro- and anti-inflammatory cytokines such as IL-1 $\beta$ and TGF- $\beta$ in CCB-GO patients. These factors are involved in initiation and progression of GO as well as periodontitis.

\section{Keywords}

\section{Gingival Overgrowth, Gingival Crevicular Fluid, Multiplex Bead Immunoassay, Periodontitis}

\section{Introduction}

Drug-induced gingival overgrowth (GO), a side effect of calcium channel blockers (CCB), immune suppressors, and anti-seizure drugs, is characterized by fibrosis with inflammation of the gingivae. In particular, CCB-GO has been increasing in Japan as well as internationally due to the rise in the use of medication in patients with hypertension. CCB such as nifedipine and amlodipine have been widely used to treat hypertension and/or angina. GO has been observed in $6 \%-30 \%$ of patients taking these drugs [1] [2]. Multiple risk factors for CCB-GO have been suggested including age, sex, genetic pre-disposition, duration of therapy, gingival inflammation, and concomitant medications, such as cyclosporine A [3].

GO results from the deposition of excessive extracellular matrix, reflecting both overproduction of collagen and reduced levels of collagen breakdown [4]-[6]. We have previously reported that matrix metalloproteinases (MMPs) and tissue inhibitors of metalloproteinases (TIMPs) play a role in the pathogenesis of periodontitis [7]-[10] as well as GO [11] [12] by controlling extracellular matrix metabolism.

We have also reported the specific transcription/transcriptome in GO-affected gingival tissues with microarray analyses [13]. Oral hygiene may play a key role, as it exacerbates the expression of drug-induced GO. In a particular clinical case of GO, it is often observed gingival inflammation with deep-pocket depth as well as periodontitis, sharing common characteristics including patient age and oral hygiene levels [14].

Periodontitis is diagnosed almost entirely based on an array of clinical attachment level (CAL), bleeding on probing, and radiographic findings. However, probing pocket depth (PPD) and CAL measurements using periodontal probes and radiographic bone levels provide information about past periodontal tissue destruction but do not elucidate the current state of disease activity or prognosis. Therefore, identification of a method for predicting the progression of periodontal diseases as well as GO or at least for declaring the current state of disease activity is necessary. Numerous previous studies have evaluated the association between periodontal diseases and factors found in gingival crevicular fluid (GCF) [15] [16]. Identification of biomarkers in GCF is considered important for the diagnosis and treatment of periodontal diseases [17] [18]. Thus, the relationships between the subgingival microbiota and host mediators should be examined by collecting subgingival biofilm and GCF samples from the same periodontal sites. Only a few investigators have attempted to measure the levels of GCF biomarkers and microbiological data obtained from the same sites [19] [20].

Recently, multiplex immunoassays have been used for samples with limited volumes such as GCF. We also previously reported the simultaneous, successful detection of levels of various types of cytokines and chemokines in GCF from periodontitis patients by using multiplex immunoassays [15].

The aim of the present study was to simultaneously quantify the levels of 14 biomarkers in GCF samples from sites with both GO and chronic periodontitis (CP) by multiplex immunoassays [15]. In addition, we examined the relationship between the subgingival microbiota and GCF biomarkers before and after therapy.

\section{Materials \& Methods}

\subsection{Study Design}

Eleven GO patients (10 males and 1 female, mean \pm SD: age: $64.4 \pm 14.0$ years) who had been systemically prescribed a CCB, either amlodipine or nifedipine, for hypertension treatment for at least 12 months were recruited. Before (baseline) and 4 weeks after initial periodontal treatments including plaque control, scaling, and root planing, GCF samples were taken from two sites per patient: GO-affected and periodontitis-affected peri- 
odontal pockets. GO sites were selected as those that were at least GO grade 2 according to GO grades defined by Eva Ingles et al. [21].

Informed consent was obtained prior to the study from all subjects in accordance with the Helsinki Declaration, and the study protocol was approved by the Institutional Review Board of the Faculty of Dentistry, Niigata University. All patients were told that they had the right to withdraw from the study at any time.

\subsection{Clinical Assessments}

A thorough medical and drug history was obtained for each patient. Smoking habits were also recorded (number of cigarettes/day, years of smoking). For more than 1 month before the study, all subjects received standard oral hygiene instructions and underwent full-mouth supragingival scaling and eventually showed a plaque control record of $<20 \%$. One week before the start of the study, a full-mouth periodontal examination was performed. The following clinical parameters were recorded: PPD, CAL, and bleeding on probing. They were recorded at six sites per tooth (mesiobuccal, buccal, distobuccal, mesiolingual, lingual, and distolingual) with a periodontal probe (CP-12 Color-Coded Probe; Hu-Friedy, Chicago, IL, USA) [22]. The sites with the most severe periodontal condition based on clinical findings were selected for sampling.

\subsection{Sample Collection}

A GO (PPD $\geq 5 \mathrm{~mm}$ ) and a CP site (PPD $\geq 4 \mathrm{~mm}$ ) were sampled in each subject. Before sampling, the individual tooth site was isolated with cotton rolls, supragingival plaque was removed carefully, and the site was gently air-dried with an air syringe. Subgingival plaque samples were taken before GCF sampling. The subgingival plaque samples were collected using paper points (10 $\mathrm{s} \times$ two times), and the samples were placed in separate microcentrifuge tubes. Then, paper strips were placed into the pocket until mild resistance was sensed and left in place for $30 \mathrm{~s}$ to obtain GCF samples. Samples visually contaminated with blood or diluted with saliva during sampling were discarded. The procedure was repeated four times at the same site using new strips each time. After recording the measurements, they were converted into actual GCF volumes using a calibration graph. The GCF samples were placed in tubes with a transport medium containing $200 \mu \mathrm{l}$ phosphate-buffered saline without calcium chloride or magnesium chloride and with $0.5 \%$ bovine serum albumin. After shaking for 15 min, the eluates were centrifuged for $10 \mathrm{~min}$ at $12,000 \times \mathrm{g}$ to remove plaque and cellular elements, and the strips were removed. The samples were frozen at $-80^{\circ} \mathrm{C}$ until further analysis.

The multiplex bead immunoassay used in this study allows a more comprehensive assessment of mediators within a single sample and is efficient and cost effective compared with traditional methods. The results obtained with the multiplex bead immunoassay were not compared with other conventional assays, such as an enzyme-linked immunoassay (ELISA). However, a recent study comparing 14 different immunoassays with different detection platforms (including a traditional solid-phase sandwich ELISA and immunobead-based assays using the fluidics Luminexfluorometry platform) reported interassay disparities as well as significant interlaboratory variation, suggesting that studies using a given assay cannot be directly extrapolated or compared with studies utilizing another assay [15]. This pilot study used a multiplex antibody capture and detection platform to quantitatively assess the effects of initial periodontal therapy on a comprehensive panel of cytokines and MMPs in GCF.

\subsection{Cytokine and Chemokine Analysis}

The following cytokines and chemokines, which are involved in inflammation and fibrosis, were selected as candidate GCF biomarkers according to our previous study [14] and analyzed: interleukin (IL)- $1 \alpha$, IL-1 $\beta$, IL-6, IL-8, MMP-1, MMP-3, MMP-12, MMP-13, transforming growth factor (TGF)- $\beta$, intercellular adhesion molecule (ICAM)-1, tumor necrosis factor (TNF)- $\alpha$, basic fibroblast growth factor (FGF-b), platelet-derived growth factor (PDGF)-bb, and vascular endothelial growth factor (VEGF)-A. The levels in GCF (pg/30 s $\times$ four times) were determined using a commercial multiplexed fluorescent bead-based immunoassay (Panomics ${ }^{\circledR}$, Fremont, CA, USA) in a Bio-Rad instrument (Bio-Rad Laboratories, Hercules, CA, USA).

\subsection{Bacterial Analysis}

Two paper points were collected in a sterile plastic tube and stored at $-30^{\circ} \mathrm{C}$. Universal primers targeting the $16 \mathrm{~S}$ 
ribosomal RNA gene, which is expressed and conserved in many types of bacteria, were used to estimate the total number of bacteria [23]. The presence and quantity of Porphyromonas gingivalis, (P. gingivalis), Tannerella forsythia (T. forsythia), and Treponema denticola (T. denticola) from the subgingival plaque were determined using real-time PCR analysis according to the method reported by Lyons et al. [24]. The number of bacteria was represented by the estimated number of cells per $10 \mu \mathrm{l}$ plaque suspension. Each ratio was calculated as the number of each type of bacteria to the total bacterial counts.

\subsection{Statistical Analysis}

Total cytokine levels (pg/30 s $\times$ four times; concentration of biomarkers in GCF collected four times) were analyzed and reported for each cytokine. Non-parametric approaches were used based on the distribution of the data. The statistical significance of the differences in clinical parameters, GCF volumes, and cytokine and chemokine levels in the GCF samples between the two groups was determined using the Mann-Whitney $U$ test. In each case, the level of significance was set at $\mathrm{P}<0.05$. The correlations between pairs of biomarkers were assessed using the Spearman correlation relationship.

\section{Results}

The clinical parameters of the GO and CP sites selected for sampling are shown in Table 1. The GO grade and the PPD in the GO group were significantly decreased after initial periodontal treatment. In addition, the PPD, CAL, and gingival index in the CP group were also significantly decreased.

In total, 12 of the 14 evaluated biomarkers were detectable within the range of the assay and were present in both GO and CP sites (Table 2). Two of the 14 biomarkers (FGF-b, PDGF-bb) were partially or completely undetectable because their levels were too low for detection by the assay.

The markers detectable in both GO and CP sites were: the cell adhesion factor, ICAM-1; the cell growth factor, VEGF-A; and the inflammatory factors, MMP-1, MMP-3, MMP-12, MMP-13, IL-1 $\alpha$, IL-1 $\beta$, IL-6, IL-8, TGF- $\beta$, and TNF- $\alpha$ (Table 2). IL-1 $\beta$ and TGF- $\beta$ in the GO group were significantly decreased after adjustment for multiple comparisons using the Wilcoxon signed-rank test $(\mathrm{P}<0.05)$. No significant differences were found for any of the biomarkers in the CP group. No significant differences in biomarkers were observed at baseline with intragroup comparison (Table 2).

At baseline, IL-1 $\alpha, \mathrm{IL}-1 \beta$, and IL-8 levels in GO sites were relatively higher than in CP sites. However, no significantly different markers were detected between before treatment for GO and before treatment for CP. The correlations between pairs of protein levels are displayed in Table 3. Thirteen of 66 possible combinations showed a tendency for a strong correlation: IL- $1 \alpha /$ IL- $1 \beta$, IL-1 $\beta /$ IL-8, IL-1 $\beta /$ MMP-3, IL-1 $\beta /$ TGF- $\beta$, IL-6/MMP-1, IL-6/MMP-3, IL-6/TNF- $\alpha$, IL-8/MMP-3, IL-8/TNF- $\alpha$, MMP-1/MMP-3, MMP-1/TGF- $\beta$, MMP-1/TNF- $\alpha$, and MMP-3/TGF- $\beta$ (Table 3 ).

Table 4 shows inter- and intragroup comparisons of subgingival bacterial counts. The T. forsythia ratio (to

Table 1. Clinical assessments in periodontitis patients before and 4 weeks after treatments (mean \pm SD).

\begin{tabular}{|c|c|c|c|c|c|c|}
\hline & \multicolumn{2}{|c|}{ GO } & \multicolumn{4}{|c|}{$\mathrm{CP}$} \\
\hline & Baseline & After & P-value & Baseline & After & P-value \\
\hline GO Grade & $2.1 \pm 0.7$ & $1.4 \pm 0.7$ & 0.03 & - & - & \\
\hline PPD (mm) & $6.0 \pm 1.4$ & $4.0 \pm 1.7$ & 0.02 & $5.0 \pm 0.7$ & $3.0 \pm 1.0$ & 0.01 \\
\hline $\mathrm{CAL}(\mathrm{mm})$ & $5.0 \pm 1.1$ & $4.0 \pm 1.5$ & 0.23 & $5.0 \pm 1.1$ & $4.0 \pm 1.1$ & 0.03 \\
\hline GI & $1.0 \pm 0.5$ & $1.0 \pm 0.6$ & 0.29 & $1.0 \pm 0.6$ & $0.0 \pm 0.5$ & 0.03 \\
\hline BOP (\% positive) & $54.5 \pm 52.2$ & $36.4 \pm 50.5$ & 0.32 & $36.4 \pm 50.5$ & $27.3 \pm 46.7$ & 0.99 \\
\hline Mo & $0.2 \pm 0.4$ & $0.2 \pm 0.4$ & 0.99 & $0.0 \pm 0.0$ & $0.0 \pm 0.0$ & 0.99 \\
\hline BL (\%) & $27.5 \pm 34.3$ & - & & $15.8 \pm 21.5$ & - & \\
\hline
\end{tabular}

Before vs. after: Wilcoxon signed-rank test; level of significance, $\mathrm{P}<0.05$. GO grade defined by Ingles et al. (New clinical index for drug-induced gingival overgrowth. Quintessence Int. 1999; 7: 467-73). PPD: Probing pocket depth; CAL: Clinical attachment level; GI: Gingival index; BOP: Bleeding on probing; Mo: Mobility of tooth; BL: bone loss by radiographic examination. 
Table 2. Protein levels (pg/ml) of GCF biomarkers before and 4 weeks after treatment (median IQR).

\begin{tabular}{|c|c|c|c|c|c|c|c|}
\hline & \multicolumn{3}{|c|}{ GO } & \multicolumn{3}{|c|}{$\mathrm{CP}$} & \multirow{2}{*}{$\begin{array}{c}{ }^{*} \text { Intragroup } \\
\text { comparison at } \\
\text { baseline }\end{array}$} \\
\hline & Baseline & After & P-value & Baseline & After & P-value & \\
\hline IL-1 $\alpha$ & 799.1 [1191.2] & 683.4 [629.7] & 0.25 & 332.7 [795.7] & 370.7 [607.1] & 0.48 & 0.38 \\
\hline IL-1 $\beta$ & $73.9[120.0]$ & 53.8 [68.2] & 0.02 & $46.3[78.8]$ & 23.6 [33.6] & 0.79 & 0.18 \\
\hline IL-6 & $2.0[2.1]$ & $1.2[1.7]$ & 0.21 & $1.8[3.2]$ & $1.8[0.6]$ & 0.42 & 0.67 \\
\hline IL-8 & 218.8 [155.0] & 222.0 [112.7] & 0.66 & 161.1 [171.8] & $106.5[94.1]$ & 0.33 & 0.97 \\
\hline ICAM-1 & 2007.3 [2856.1] & 2157.3 [557.7] & 0.42 & 2255.0 [3246.7] & 2038.8 [2310.5] & 0.92 & 0.72 \\
\hline MMP-1 & 71.0 [92.5] & $108.1[76.8]$ & 0.48 & $69.3[52.0]$ & 63.6 [90.2] & 0.66 & 0.92 \\
\hline MMP-3 & 214.4 [395.9] & 206.4 [326.5] & 0.48 & 202.5 [178.1] & 91.4 [108.7] & 0.13 & 0.20 \\
\hline MMP-12 & 142.1 [338.6] & 322.5 [370.9] & 0.13 & $231.2[377.0]$ & 109.1 [162.3] & 0.51 & 0.40 \\
\hline MMP-13 & 611.6 [3143.6] & $1444.0[2742.7]$ & 0.79 & 1073.4 [1780.8] & 811.9 [1582.1] & 0.29 & 0.72 \\
\hline TGF- $\beta$ & $4.4[5.6]$ & $3.1[3.1]$ & 0.01 & $4.1[5.1]$ & $1.7[1.3]$ & 0.29 & 0.49 \\
\hline TNF- $\alpha$ & $1.3[0.6]$ & $1.8[1.2]$ & 0.79 & $1.8[2.4]$ & $1.4[1.2]$ & 0.88 & 0.53 \\
\hline VEGF-A & 6.6 [9.9] & $7.1[8.1]$ & 0.25 & $6.3[6.7]$ & $6.7[4.9]$ & 0.33 & 0.41 \\
\hline
\end{tabular}

before vs. after: Wilcoxon signed-rank test; level of significance, $\mathrm{P}<0.05$, GO-before vs. CP-before: Mann-Whitney U test; level of significance, $\mathrm{P}<$ 0.05 , IQR = Interquartile range; Not detectable: FGF-b, PDGF-bb; Values are the median IQR.

Table 3. Correlations between pairs of GCF biomarkers.

\begin{tabular}{|c|c|c|c|c|c|c|c|c|c|c|c|c|}
\hline Variable & IL-1 $\alpha$ & IL- $1 \beta$ & IL-6 & IL-8 & MMP-1 & MMP-3 & MMP-12 & MMP-13 & TGF- $\beta$ & TNF- $\alpha$ & VEGF- $\beta$ & ICAM-1 \\
\hline IL-1 $\alpha$ & | & 0.66 & 0.18 & 0.18 & 0.05 & 0.19 & -0.41 & -0.56 & 0.41 & -0.13 & 0.25 & -0.05 \\
\hline IL- $1 \beta$ & & | & 0.11 & 0.62 & 0.32 & 0.62 & -0.50 & -0.53 & 0.74 & 0.28 & 0.25 & -0.82 \\
\hline IL-6 & & & | & 0.55 & 0.62 & 0.67 & 0.39 & 0.22 & 0.10 & 0.69 & -0.30 & 0.10 \\
\hline IL-8 & & & & | & 0.64 & 0.82 & -0.25 & 0.11 & 0.41 & 0.63 & -0.20 & 0.25 \\
\hline MMP-1 & & & & & | & 0.72 & 0.38 & 0.37 & 0.62 & 0.62 & -0.01 & 0.36 \\
\hline MMP-3 & & & & & & I & 0.03 & -0.11 & 0.62 & 0.54 & 0.03 & 0.07 \\
\hline MMP-12 & & & & & & & | & 0.55 & -0.27 & 0.33 & -0.16 & 0.37 \\
\hline MMP-13 & & & & & & & & | & -0.54 & -0.52 & 0.10 & 0.67 \\
\hline TGF- $\beta$ & & & & & & & & & 1 & -0.11 & 0.65 & -0.31 \\
\hline TNF- $\alpha$ & & & & & & & & & & | & 0.65 & 0.50 \\
\hline VEGF- $\beta$ & & & & & & & & & & & | & -0.71 \\
\hline ICAM-1 & & & & & & & & & & & & | \\
\hline
\end{tabular}

Statistics: Spearman correlation relationship. Bold: significant correlations.

total bacteria) in the $\mathrm{CP}$ group was significantly decreased after 4 weeks compared to baseline $(\mathrm{P}<0.05)$. The intragroup comparison at baseline showed that the counts of $T$. denticola in the GO group were significantly higher than that of the CP group $(\mathrm{P}<0.05)$.

\section{Discussion}

In the present study, we demonstrated that two biomarkers, IL- $1 \beta$ and TGF- $\beta$, in GCF were significantly decreased in GO patients after initial periodontal treatment. The periodontal pathogenic bacteria $T$. denticola were significantly higher in GO patients presenting with periodontal pockets and were decreased after treatment. 
Table 4. Multiple logistic regression analysis for progression of chronic periodontitis.

\begin{tabular}{cccccccc}
\hline & \multicolumn{2}{c}{ GO } & \multicolumn{3}{c}{ CP } & \multicolumn{2}{c}{$\begin{array}{c}\text { Intragroup } \\
\text { comparison }\end{array}$} \\
\cline { 2 - 8 } & Baseline & After 4 weeks & P-value & Baseline & After 4 weeks & P-value & P-value $^{*}$ \\
\hline Total bacterial amount & $5.3(0.6)$ & $4.8(0.6)$ & 0.17 & $4.8(0.4)$ & $4.6(0.5)$ & 0.31 & 0.43 \\
P. gingivalis $\left(\log _{10}\right)$ & $0.5(1.8)$ & $0.5(0.0)$ & 0.66 & $0.50(0.0)$ & $0.50(0.0)$ & 0.08 & 0.55 \\
T. forsythia $\left(\log _{10}\right)$ & $2.50(2.9)$ & $2.10(2.5)$ & 0.37 & $2.40(2.0)$ & $0.50(1.0)$ & 0.08 & 0.21 \\
T. denticola $\left(\log _{10}\right)$ & $2.10(1.8)$ & $1.70(1.6)$ & 0.52 & $0.50(0.9)$ & $0.50(0.0)$ & 0.16 & $\mathbf{0 . 0 4}$ \\
P. gingivalis ratio (\%) & $0.00(0.0)$ & $0.00(0.0)$ & 0.66 & $0.00(0.0)$ & $0.00(0.0)$ & 0.08 & 0.66 \\
T. forsythia ratio (\%) & $0.41(0.78)$ & $0.16(0.31)$ & 0.32 & $0.08(0.29)$ & $0.00(0)$ & $\mathbf{0 . 0 4}$ & 0.35 \\
T. denticola ratio (\%) & $0.04(0.08)$ & $0.04(0.02)$ & 0.65 & $0.00(0)$ & $0.00(0)$ & 0.25 & 0.28 \\
\hline
\end{tabular}

Before vsafter: Wilcoxon signed-rank test; before-GO vsbefore-CP: Mann-Whitney U test; level of significance, $\mathrm{P}<0.05$, IQR = Interquartile range. The ${ }^{*}$ represents the intragroup comparison between baseline in GO and CP before treatments.

The levels of IL-1 $\beta$ and TGF- $\beta$ in GO-GCF were significantly decreased 4 weeks after initial conventional periodontal treatments including scaling and root planing. The decreased IL- $1 \beta$ and TGF- $\beta$ levels seemed to be associated with levels of gingival enlargement and fibrosis [25] [26].

IL-1 $\beta$ stimulates the proliferation of fibroblasts, keratinocytes, and endothelial cells and enhances the synthesis of type 1 procollagen, hyaluronate, and fibronectin [13]. As seen in periodontitis, the pathogenesis of GO is also modified by proliferation, growth factors, and MMPs [13]. Lu et al. reported that nifedipine and IL-1 $\beta$ play important roles in GO via androgen receptor up-regulation that results in collagen accumulation [27]. Similar pathogenesis was also reported in cyclosporine A- and phenytoin-induced GO [28]-[31]. Recently, GCF IL-1 $\beta$ levels in patients with CCB-GO were reported to be significantly decreased after periodontal treatment in GO patients, which is in agreement with our results. Thus, IL-1 $\beta$ may play a role in GO pathogenesis.

TGF- $\beta$ is widely recognized as a key fibrogenic cytokine [32]. TGF- $\beta 1$ is also a multifunctional cytokine with pleiotropic properties, exhibiting both pro-inflammatory and anti-inflammatory effects that modulate cell proliferation and differentiation by inhibiting the destruction of extracellular matrix proteins through the suppression of matrix-degrading proteinases [19]. Kim et al. demonstrated that nifedipine up-regulates periostin in human gingival fibroblasts in a TGF- $\beta$-dependent manner [33]. The present study demonstrated significantly decreased TGF- $\beta$ levels in GO-GCF after initial periodontal treatments, implying a similar relationship between TGF- $\beta$ levels and the degree of fibrosis in GO patients. Wright et al. also reported that subjects with high levels of TGF- $\beta$ may be at greater risk for cyclosporine-induced gingival overgrowth [19]. Findings including ours suggested that TGF- $\beta$ levels could contribute to the fibrotic changes in gingival connective tissues in patients with CCB-GO as well.

The etiology of CCB-GO appears complex, similar to periodontitis [34]. Gingival tissues of CCB-GO patients are often seen with clinically mixed features of GO and CP [14]. Correlation analysis revealed that IL-8 and MMP-3, IL- $1 \beta$ and TGF- $\beta$, and MMP-1 and MMP-3 showed strong correlations. These associations could be related to the pathogenesis of the mixed features of GO.

Multiplex immunoassays have the advantage of the capability of analyzing samples with limited volumes such as GCF. We previously reported simultaneous successful quantification of various cytokine and chemokine levels in GCF from periodontitis patients [15]. The present study compared the levels of 12 biomarkers at both GO and CP sites in each patient with GO-mixed periodontitis presenting with GO as well.

GCF biomarkers are considered to be important for clarifying the mechanism of gingiva inflammation [15]. GCF itself is considered to be important for diagnosis of periodontal diseases because inflammation of periodontal tissues can be measured in this fluid. The diagnostic potential of GCF has been extensively investigated because of the possibility of non-invasive collection and a rich array of cellular and biochemical molecules associated with disease activity [35].

However, because of the small sample volumes, evaluating the relationships among the various types of biomarkers is sometimes difficult. The present study successfully compared the levels of 12 biomarkers at both GO and CP sites in each subject with periodontitis. Our findings suggest that the cytokine expression profiles, espe- 
cially levels of IL- $1 \beta$ and TGF- $\beta$, in GCF could be markers of the GO status and are detectable with the multiplex bead immunoassay.

Gingival inflammation induced by periodontopathic bacteria increases the risk of occurrence of GO [36]-[38]. The interaction between nifedipine and bacterial lipopolysaccharide may contribute to CCB-GO [39]. Patients with GO present with increased prevalence of $P$. gingivalis, $T$. denticola, and $T$. forsythia (red complex) as well as significantly higher levels of IL- $1 \beta$ than those without GO [36]. Periodontal GO patients that carry the CD14-260 genotypes CT + TT with allelic T have higher frequencies of $P$. gingivalis, $T$. denticola, and $T$. forsythia compared to those carrying the CC genotype [40]. Counts of $P$. gingivalis and $T$. denticola in periodontal pockets of cyclosporine A-GO patients are significantly higher than those of CP patients [40]. However, our results demonstrated that only $T$. denticola was significantly higher. Certain mechanisms of drug-induced GO by periodontopathic bacteria such as T. denticola may be involved. The total bacterial counts tented to be decreased, though it did not reach the significant levels. It could be due to the individual variation of the microbial flora and the plaque control levels. According to some previous reports and the present study, CCB-GO could be effectively prevented by controlling microbial plaque factors, nevertheless CCB were continued in the patients [41]. Thus the supportive periodontal therapy (SPT) must be one of the most important ways to preventing GO recurrences.

In the future, a comprehensive study of bacterial adhesions and virulence factors, their molecular targets, and host proteases using proteomic and peptidomic approaches will likely be valuable for identifying molecular disease progression and for discriminating among the various manifestations of GO.

Consequently, the ease, efficiency, and expansiveness of multiplex technology may lead to a better understanding of not only the differences in cytokine profiles in periodontal disease and GO states but also the impact of periodontal therapy on inflammatory mediators. Periodontal pathogens may be related to the initiation and development of periodontal treatment for GO. Inflammatory cytokines (IL-1 $\beta$ and TGF- $\beta 1$ ) may also be considered contributing factors.

\section{Conclusion}

In conclusion, the significant GCF biomarkers, IL- $1 \beta$ and TGF- $\beta 1$ were detected in sites with GO periodontal pockets by multiplex bead immunoassay and decreased by periodontal initial treatments. Simultaneously the presence of $T$. denticola, could also be involved in the initiation and the progression of peiodontitis presenting with GO.

\section{Acknowledgements}

This work was supported by a Grant-in-Aid for Scientific Research (No. 26861809) from the Japanese Ministry of Education, Culture, Sports, Science and Technology of Japan.

\section{References}

[1] Trackman, P.C. and Kantarci, A. (2004) Connective Tissue Metabolism and Gingival Overgrowth. Critical Reviews in Oral Biology \& Medicine, 15, 165-175. http://dx.doi.org/10.1177/154411130401500305

[2] Ellis, J.S., Seymour, R.A., Steele, J.G., Robertson, P., Butler, T.J. and Thomason, J.M. (1999) Prevalence of Gingival Overgrowth Induced by Calcium Channel Blockers: A Community-Based Study. Journal of Periodontology, 70, 63-67. http://dx.doi.org/10.1902/jop.1999.70.1.63

[3] Bostanci, N., Ilgenli, T., Pirhan, D.C., Clarke, F.M., Marcenes, W., Atilla, G., Hughes, F.J. and McKay, I.J. (2006) Relationship between IL-1A Polymorphisms and Gingival Overgrowth in Renal Transplant Recipients Receiving Cyclosporine A. Journal Clinical of Periodontology, 33, 771-778. http://dx.doi.org/10.1111/j.1600-051X.2006.00990.x

[4] Rostock, M.H., Fry, H.R. and Turner, J.E. (1986) Severe Gingival Overgrowth Associated with Cyclosporine Therapy. Journal of Periodontology, 57, 294-299. http://dx.doi.org/10.1902/jop.1986.57.5.294

[5] Seymour, R.A., Thomason, J.M. and Ellis, J.S. (1996) The Pathogenesis of Drug-Induced Gingival Overgrowth. Journal Clinical of Periodontology, 23, 165-175. http://dx.doi.org/10.1111/j.1600-051X.1996.tb02072.x

[6] Barak, S., Engelberg, I.S. and Hiss, J. (1987) Gingival Hyperplasia Caused by Nifedipine. Histopathologic Findings. Journal of Periodontology, 58, 639-642. http://dx.doi.org/10.1902/jop.1987.58.9.639

[7] Kubota, T., Itagaki, M., Morozumi, T., Maruyama, S., Nakasone, N., Shimizu, T., Takashi, S. and Hiromasa, Y. (2007) A Case Report of Multiple-Drug-Induced Gingival Overgrowth with TIMP-3 Over-Expression. Oral Medicine \& Pa- 
thology, 12, 141-148. http://dx.doi.org/10.3353/omp.12.141

[8] Kubota, T., Nomura, T., Takahashi, T. and Hara, K. (1996) Expression of mRNA for Matrix Metalloproteinases and Tissue Inhibitors of Metalloproteinases in Periodontitis-Affected Human Gingival Tissue. Archives of Oral Biology, 41, 253-262. http://dx.doi.org/10.1016/0003-9969(95)00126-3

[9] Kubota, T., Matsuki, Y., Nomura, T. and Hara, K. (1997) In Situ Hybridization Study on Tissue Inhibitors of Metalloproteinases (TIMPs) mRNA-Expressing Cells in Human Inflamed Gingival Tissue. Journal of Periodontal Research, 32, 467-472. http://dx.doi.org/10.1111/j.1600-0765.1997.tb00559.x

[10] Dannewitz, B., Edrich, C., Tomakida, P., Kohl, A., Gabbert, O., Eickholz, P. and Steinberg, T. (2006) Elevated Gene Expression of MMP-1, MMP-10, and TIMP-1 Reveal Changes of Molecules Involved in Turn-Over of Extracellular Matrix in Cyclosporine-Induced Gingival Overgrowth. Cell and Tissue Research, 325, 513-522.

http://dx.doi.org/10.1007/s00441-006-0200-X

[11] Barclay, S., Thomason, J.M., Idle, J.R. and Seymour, R.A. (1992) The Incidence and Severity of Nifedipine-Induced Gingival Overgrowth. Journal Clinical of Periodontology, 19, 311-314. http://dx.doi.org/10.1111/j.1600-051X.1992.tb00650.x

[12] Yamada, H., Nishimura, F., Naruishi, K., Chou, H.H., Takashiba, S., Albright, G.M., Nares, S., Iacopino, A.M. and Murayama, Y. (2000) Phenytoin and Cyclosporine A Suppress the Expression of MMP-1, TIMP-1, and Cathepsin L, but Not Cathepsin B in Cultured Gingival Fibroblasts. Journal of Periodontology, 71, 955-960. http://dx.doi.org/10.1902/jop.2000.71.6.955

[13] Shimizu, T., Kubota, T., Nakasone, N., Abe, D., Morozumi, T. and Yoshie, H. (2011) Microarray and Quantitative RT-PCR Analyses in Calcium-Channel Blockers Induced Gingival Overgrowth Tissues of Periodontitis Patients. Archives of Oral Biology, 56, 277-284. http://dx.doi.org/10.1016/j.archoralbio.2010.10.002

[14] Nakasone, N., Kubota, T., Hoshino, C., Nohno, K., Itagaki, M., Shimizu, T. and Yoshie, H. (2009) Differential Gene and Protein Expression of Tissue Inhibitors of Metalloproteinases (TIMP)-3 and TIMP-4 in Gingival Tissues from Drug Induced Gingival Overgrowth. Archives of Oral Biology, 54, 634-641. http://dx.doi.org/10.1016/j.archoralbio.2009.04.002

[15] Shimada, Y., Tabeta, K., Suqita, N. and Yoshie, H. (2013) Profiling Biomarkers in Gingival Crevicular Fluid Using Multiplex Bead Immunoassay. Archives of Oral Biology, 58, 724-730. http://dx.doi.org/10.1016/j.archoralbio.2012.11.012

[16] Offenbacher, S., Barros, S., Mendoza, L., Mauriello, S., Preisser, J., Moss, K., de Jager, M. and Aspiras, M. (2010) Changes in Gingival Crevicular Fluid Inflammatory Mediator Levels during the Induction and Resolution of Experimental Gingivitis in Humans. Journal Clinical of Periodontology, 37, 324-333. http://dx.doi.org/10.1111/j.1600-051X.2010.01543.x

[17] Champagne, C.M., Buchanan, W., Reddy, M.S., Preisser, J.S., Beck, J.D. and Offenbacher, S. (2003) Potential for Gingival Crevice Fluid Measures as Predictors of Risk for Periodontal Diseases. Periodontology 2000, 31, 167-180. http://dx.doi.org/10.1034/j.1600-0757.2003.03110.x

[18] Loos, B.G. and Tjoa, S. (2005) Host-Derived Diagnostic Markers for Periodontitis: Do They Exist in Gingival Crevice Fluid? Periodontology 2000, 39, 53-72. http://dx.doi.org/10.1111/j.1600-0757.2005.00129.x

[19] Teles, R., Sakellari, D., Teles, F., Konstantinids, A., Kent, R., Socransky, S. and Haffajee, A. (2010) Relationships among Gingival Crevicular Fluid Biomarkers, Clinical Parameters of Periodontal Disease, and the Subgingival Microbiota. Journal of Periodontology, 81, 89-98. http://dx.doi.org/10.1902/jop.2009.090397

[20] Wright, H.J., Chapple, I.L., Blair, F. and Matthews, J.B. (2004) Crevicular Fluid Levels of TGF Beta 1 in Drug-Induced Gingival Overgrowth. Archives of Oral Biology, 49, 421-425. http://dx.doi.org/10.1016/j.archoralbio.2003.09.002

[21] Inglés, E., Rossmann, J.A. and Caffesse, R.G. (1999) New Clinical Index for Drug-Induced Gingival Overgrowth. Quintessence International, 7, 467-473.

[22] Morozumi, T., Kubota, T., Abe, D., Shimizu, T., Komatsu, Y. and Yoshie, H. (2010) Effects of Irrigation with an Antiseptic and Oral Administration of Azithromycin on Bacteremia Caused by Scaling and Root Planing. Journal of Periodontology, 81, 1555-1563. http://dx.doi.org/10.1902/jop.2010.100163

[23] Ashimoto, A., Chen, C., Bakker, I. and Slots, J. (1996) Polymerase Chain Reaction Detection of 8 Putative Periodontal Pathogens in Subgingival Plaque of Gingivitis and Advanced Periodontitis Lesions. Oral Microbiology and Immunology, 11, 266-273. http://dx.doi.org/10.1111/j.1399-302X.1996.tb00180.x

[24] Lyons, S.R., Griffen, A.L. and Leys, E.J. (2000) Quantitative Real-Time PCR for Porphyromonas Gingivalis and Total Bacteria. Journal of Clinical Microbiology, 38, 2362-2365.

[25] Kim, S.S., Jackson-Boeters, L., Darling, M.R., Rieder, M.J. and Hamilton, D.W. (2013) Nifedipine Induces Periostin Expression in Gingival Fibroblasts through TGF-Beta. Journal of Dental Research, 92, 1022-1028. 
http://dx.doi.org/10.1177/0022034513503659

[26] Johnson, R.B., Zebrowski, E.J. and Dai, X. (2000) Synergistic Enhancement of Collagenous Protein Synthesis by Human Gingival Fibroblasts Exposed to Nifedipine and Interleukin-1-Beta in Vitro. Journal of Oral Pathology \& Medicine, 29, 8-12. http://dx.doi.org/10.1034/j.1600-0714.2000.290102.x

[27] Lu, H.K., Tseng, C.C., Lee, Y.H., Li, C.L. and Wang, L.F. (2010) Flutamide Inhibits Nifedipine- and Interleukin-1 Beta-Induced Collagen Overproduction in Gingival Fibroblasts. Journal of Periodontal Research, 45, 451-457.

[28] Salman, B.N., Vahabi, S., Movaghar, S.E. and Mahjour, F. (2013) Proliferative and Inductive Effects of Cyclosporine A on Gingival Fibroblast of Child and Adult. Journal of Dental Research, 10, 52-58.

[29] Johanson, M., Zhao, X.R., Huynh-Ba, G. and Villar, C.C. (2013) Matrix Metalloproteinases, Tissue Inhibitors of Matrix Metalloproteinases, and Inflammation in Cyclosporine A-Induced Gingival Enlargement: A Pilot in Vitro Study Using a Three-Dimensional Model of the Human Oral Mucosa. Journal of Periodontology, 84, 623-640. http://dx.doi.org/10.1902/jop.2012.120224

[30] Andriamanalijaona, R., Benateau, H., Barre, P.E., Boumediene, K., Labbe, D., Compere, J.F. and Pujol, J.P. (2006) Effect of Interleukin-1Beta on Transforming Growth Factor-Beta and Bone Morphogenetic Protein-2 Expression in Human Periodontal Ligament and Alveolar Bone Cells in Culture: Modulation by Avocado and Soybean Unsaponifiables. Journal of Periodontology, 77, 1156-1166. http://dx.doi.org/10.1902/jop.2006.050356

[31] Sattari, M., Fathiyeh, A., Gholami, F., Darbandi Tamijani, H. and Ghatreh Samani, M. (2011) Effect of Surgical Flap on IL- $1 \beta$ and TGF- $\beta$ Concentrations in the Gingival Crevicular Fluid of Patients with Moderate to Severe Chronic Periodontitis. Iranian Journal of Immunology, 8, 20-26.

[32] Radwan-Oczko, M., Boratynska, M., Zietek, M. and Dobosz, T. (2008) Transforming Growth Factor- $\beta 1$ Gene Expression and Cyclosporine A-Induced Gingival Overgrowth: A Pilot Study. Journal of Clinical Periodontology, 35, 371378. http://dx.doi.org/10.1111/j.1600-051X.2008.01216.X

[33] Kim, S.S., Jackson-Boeters, L., Darling, M.R., Rieder, M.J. and Hamilton, D.W. (2013) Nifedipine Induces Periostin Expression in Gingival Fibroblasts through TGF-Beta. Journal of Dental Research, 92, 1022-1028. http://dx.doi.org/10.1177/0022034513503659

[34] Kornman, K.S. (2008) Mapping the Pathogenesis of Periodontitis: A New Look. Journal of Periodontology, 79, 15601568. http://dx.doi.org/10.1902/jop.2008.080213

[35] Brown, J.M., Watanabe, K., Cohen, R.L. and Chambers, D.A. (1995) Molecular Characterization of Plasminogen Activators in Human Gingival Crevicular Fluid. Archives of Oral Biology, 40, 839-845. http://dx.doi.org/10.1016/0003-9969(95)00049-U

[36] Pernu, H.E., Pernu, L.M., Huttunen, K.R., Nieminen, P.A. and Knuuttila, M.L. (1992) Gingival Overgrowth among Renal Transplant Recipients Related to Immunosuppressive Medication and Possible Local Background Factors. Journal of Periodontology, 63, 548-553. http://dx.doi.org/10.1902/jop.1992.63.6.548

[37] Guo, J., Wang, W., Yao, L. and Yan, F. (2008) Local Inflammation Exacerbates Cyclosporine A-Induced Gingival Overgrowth in Rats. Inflammation, 31, 399-407. http://dx.doi.org/10.1007/s10753-008-9091-1

[38] Fu, E., Nieh, S. and Wikesjö, U.M. (1997) The Effect of Plaque Retention on Cyclosporine-Induced Gingival Overgrowth in Rats. Journal of Periodontology, 68, 92-98. http://dx.doi.org/10.1902/jop.1997.68.1.92

[39] Takeuchi, R., Matsumoto, H., Akimoto, Y. and Fujii, A. (2011) Reduction in Lipopolysaccharide-Induced Apoptosis of Fibroblasts Obtained from a Patient with Gingival Overgrowth during Nifedipine-Treatment. Archives of Oral Biology, 56, 1073-1080. http://dx.doi.org/10.1016/j.archoralbio.2011.03.006

[40] Gong, Y., Bi, W., Cao, L., Yang, Y., Chen, J. and Yu, Y. (2013) Association of CD14-260 Polymorphisms, Red-Complex Periodontopathogens and Gingival Crevicular Fluid Cytokine Levels with Cyclosporine A-Induced Gingival Overgrowth in Renal Transplant Patients. Journal of Periodontal Research, 48, 203-212. http://dx.doi.org/10.1111/j.1600-0765.2012.01521.x

[41] Akiyama, S., Amano, A., Kato, T., Takada, Y., Kimura, K.R. and Morisaki, L. (2006) Relationship of Periodontal Bacteria and Prophyromonas gingivalis fimA Variations with Phenytoin-Induced Gingival Overgrowth. Oral Diseases, 12, 51-56. http://dx.doi.org/10.1111/j.1601-0825.2005.01157.x 